\title{
Image Simulation of Gold Cluster Detection in TEM and STEM
}

\author{
J. Wall
}

Biology Dept., Brookhaven National Laboratory, Upton N.Y. 11973

Image simulation plays a critical role in materials science microscopy due to the high likelihood that atoms will be found in ordered arrangements, thereby giving coherent scattering. The "random" arrangement of atoms in most biological specimens suggests that simpler approximations (weak phase object) might be adequate. We have investigated this question using large colledions of atoms and the multi-slice software of Kirkland ${ }^{1}$. In this example we compare TEM phase contrast with STEM dark field, both at $200 \mathrm{keV}$, for detection of undecagold clusters in ice.

The only assumption required is that the specimen is composed of atoms. The calculation gives the image at infinite dose and absolute zero temperature with no incident energy spread, no inelastic scattering, radiation damage, vibration, drift, charging, or other parasitic effects. The effects of finite dose, etc. can be added back if desired. A basic feature of the calculation is that the intensities are normalized as a fraction of the incident dose, making comparison to experiment fairly straightforward. We can also compare STEM to TEM for imaging the same specimen.

The practical barrier to realistic simulations is the number of atoms needed to describe a biological complex with enough of the surrounding substrate and ice to permit reasonable defocus values. We typically use 25.6 × $25.6 \times 25.6 \mathrm{~nm}$ containing roughly450,000 atoms. The atomic coordinates of known structures such as TMV, apoferritin, groEL or various ribosomal particles can be obtained from the Protein Data Bank. This may require a symmetry operation to pack subunits into the overall structure. We use a random film generator to place carbon atoms with a nearest neighbor distance $>0.2 \mathrm{~nm}$ to a desired thickness and random water generator to place oxygen atoms in the remaining spaces with a nearest neighbor distance $>0.29 \mathrm{~nm}$. At present we are not induding hydrogen atoms, although they are expected to have a substantial effect on the phase contrast image.

For the simulation shown, we have used 10 undecagold clusters at random locations with random orientations. The multi-slice calculation accepts a file containing a list giving: atomic number, $\mathrm{x}, \mathrm{y}, \mathrm{z}$ coordinates, temperature factor and occupancy for each atom. In this example the intermediate file is $20 \mathrm{Mb}$ in size. The multi-slice procedure is carried out in three steps: 1) computation of the wave function entering the specimen, 2) propagation, wave optically, through the specimen in a series of slices, each thin enough to be treated linearly and 3) transformation of the emerging wave function to either the diffraction or the image plane. In the TEM case, the incident wave can be plane (coherent illumination) or partially coherent and the imaging includes the effects of aberrations \& defocus. In the STEM case, the incident wave is from the probeforming lens, including aberrations $\&$ defocus and the emerging wave forms a convergent beam electron diffraction (cbed) pattern at the detector plane. The STEM signals are computed by integrating the intensity distribution over those areas of the cbed pattern falling on the various detectors. For practical purposes, the STEM signal can be computed by summing the signals from the individual atoms incoherently. Propagation through the thin specimen slices is done by computing the net potential distribution from all the atoms in that slice, taking into account nuclear charge and screening for each atom. 

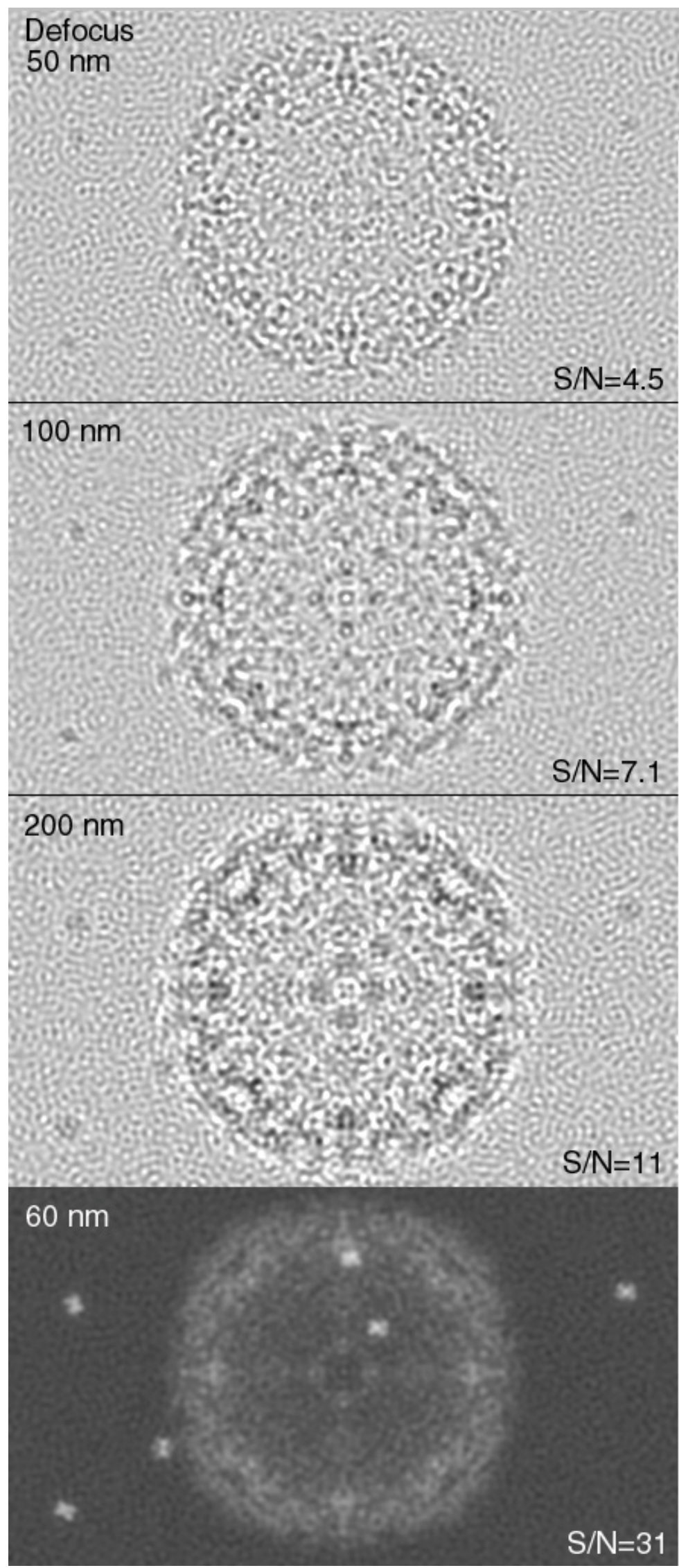

The example shows the potential advantage of STEM for detection of gold clusters in moderately thin ice. In the TEM the clusters in the background are detectable, but those near the apoferritin are not. The effect of finite dose is relatively straightforward to include, since the image intensities are calculated as a percentage of the incident dose.

An interesting feature of the TEM images is the phase grain in the background. This adds a random phase error to the phase contrast image. We are investigating the effect of this on image reconstruction.

Figure 1. Simulated image of apoferritin and ten undecagold clusters embedded in $20 \mathrm{~nm}$ thick amorphous ice imaged at infinite dose. Accelerating voltage $=200$ $\mathrm{keV}, \mathrm{Cs}=1.4 \mathrm{~mm}$ and defocus is given. Upper three images are bright field phase contrast in TEM and lower image is dark field STEM (incoherent large angle scattering). The signal to noise ratio $(\mathrm{S} / \mathrm{N})$ was measured for the three isolated gold clusters in the ice and another four out of the field of view as compared to randomly selected points in the background region. The intensity of the STEM image is not on the same scale, to illustrate the visibility of the gold clusters.

\section{Reference}

1. Kirkland, E. J. (1998). Advanced computing in electron microscopy. Plenum, New York. 